\title{
Insight into lithium-sulfur batteries: Elementary kinetic modeling and impedance simulation
}

\author{
David N. Fronczek ${ }^{\mathrm{a}, \mathrm{b}}$, Wolfgang G. Bessler ${ }^{\mathrm{a}, \mathrm{c}, *}$ \\ ${ }^{a}$ German Aerospace Center (DLR), Institute of Technical Thermodynamics, Pfaffenwaldring 38-40, 70569 Stuttgart, Germany \\ ${ }^{b}$ Helmholtz Institute Ulm for Electrochemical Energy Storage (HIU), Albert-Einstein-Allee 11, 89069 Ulm, Germany \\ ${ }^{c}$ Offenburg University of Applied Sciences, Badstrasse 24, 77652 Offenburg, Germany
}

\begin{abstract}
We present a model of the lithium/sulfur $(\mathrm{Li} / \mathrm{S})$ battery based on a multi-step, elementary sulfur reduction mechanism including dissolved polysulfide anions. The model includes a description of the evolution of solid phases in the carbon/sulfur composite cathode as well as multi-component $\left(\mathrm{Li}^{+}, \mathrm{PF}_{6}^{-}, \mathrm{S}_{8}, \mathrm{~S}_{8}^{2-}, \mathrm{S}_{6}^{2-}, \mathrm{S}_{4}^{2-}, \mathrm{S}_{2}^{2-}, \mathrm{S}^{2-}\right)$ mass and charge transport in the liquid electrolyte. The chemical reaction mechanism consists of a $\mathrm{Li} / \mathrm{Li}^{+}$oxidation reaction at the anode and a six-step polysulfide reduction mechanism at the cathode. The modeling framework allows for the simulation of charge and discharge profiles as well as electrochemical impedance spectra. The latter are obtained via a voltage step/current relaxation technique based on the physicochemical model without the need for applying equivalent circuit models. The results indicate that the discharge behavior of this $\mathrm{Li} / \mathrm{S}$ cell is governed by the presence of solid reactant and product phases in exchange with the dissolved polysulfide anions. The first and last stages of the discharge are characterized by the presence of solid $\mathrm{S}_{8}$ and $\mathrm{Li}_{2} \mathrm{~S}$, respectively, while the intermediate stage corresponds to a situation where all chemical compounds are dissolved in the electrolyte.
\end{abstract}

Keywords: lithium-sulfur battery, modeling, simulation, electrochemical impedance spectroscopy, EIS, polysulfides PACS: $82.20 . \mathrm{Wt}$

\section{Introduction}

The lithium-sulfur $(\mathrm{Li} / \mathrm{S})$ battery is a promising electrochemical cell for mobile energy storage $[1,2,3]$. The combination of high gravimetric energy density and environmentally benign and affordable materials is unparalleled among prospective battery materials. However, the complex redox chemistry of sulfur, which is forming a large number of soluble intermediates, presents a major challenge to understanding and mastering $\mathrm{Li} / \mathrm{S}$ electrochemistry.

Although considerable research activities have been recently carried out in order to investigate the reactions taking place during discharge and charge $[4,5,6,7,8,9]$, the precise reaction mechanism still is a subject of debate and may not even be the same for different electrolytes. A discussion of previously proposed mechanisms can be found in Refs. $[9,10,11]$.

The poor electronic conductivity of both $\mathrm{S}_{8}$ and $\mathrm{Li}_{2} \mathrm{~S}$, the dissolution and precipitation of solid phases and the unstable solid-electrolyte interface, which is continuously re-formed during cycling, present major challenges to the advancement of the $\mathrm{Li} / \mathrm{S}$ cell. Physically-based models can

\footnotetext{
*Corresponding author. Tel.: +49 781205 4653; fax: +49 781 205454653

Email address: wolfgang.bessler@hs-offenburg.de (Wolfgang G. Bessler)
}

address all of the above and provide new and deeper insight into the Li/S cell. Only few modeling studies of the $\mathrm{Li} / \mathrm{S}$ cell are published to date, the most comprehensive of which is the work of Kumaresan et al. [12]. Here, we extend the previous work of Kumaresan et al. by presenting simulation results for various discharge rates, discharge/charge cycles, as well as electrochemical impedance spectra. The simulations are based on a 1D continuum model of electrochemistry, transport, and phase management. They are carried out within our recently developed flexible framework for multi-phase management in batteries and fuel cells [13].

\section{Modeling}

\subsection{Model layout}

The geometry of the simulated cell is shown in Fig. 1. The illustration also includes the initial composition of the cathode, which is corresponding to a highly porous cell with high sulfur content in the cathode $(>80 \%$ by weight). In the directions parallel to the separator layer, the cell is assumed to be homogenous and of infinite size. This allows for the use of a $1 \mathrm{D}$ transport model in the direction of the lithium diffusion. The modeling domain consists of a lithium metal anode, a porous electrolyte-filled separator, and a composite cathode consisting of carbon, 
sulfur, lithium sulfide, and liquid electrolyte. The liquid electrolyte is in contact with all of the solid phases.

The anode is assumed to be able to supply an unlimited amount of lithium according to

$$
\mathrm{Li} \rightleftarrows \mathrm{Li}^{+}+\mathrm{e}^{-} \text {. }
$$

Its kinetics is chosen sufficiently fast, so that the anode overpotential is negligible. It is assumed that no other reaction is taking place at the anode. Because this study is focusing on the sulfur electrode, this assumption is made to simplify the interpretation of the results. Although fully protected lithium anodes are still not available, substantial progress has been made toward that goal, cf. [14]. Concerning the cathode electrochemistry, the following reactions are considered in this model, following Kumaresan et al. [12]:

$$
\begin{aligned}
\mathrm{S}_{8(\mathrm{~s})} & \rightleftarrows \mathrm{S}_{8(\mathrm{ds})} \\
1 / 2 \mathrm{~S}_{8(\mathrm{ds})}+\mathrm{e}^{-} & \rightleftarrows 1 / 2 \mathrm{~S}_{8}^{2-} \\
3 / 2 \mathrm{~S}_{8}^{2-}+\mathrm{e}^{-} & \rightleftarrows 2 \mathrm{~S}_{6}^{2-} \\
\mathrm{S}_{6}^{2-}+\mathrm{e}^{-} & \rightleftarrows 3 / 2 \mathrm{~S}_{4}^{2-} \\
1 / 2 \mathrm{~S}_{4}^{2-}+\mathrm{e}^{-} & \rightleftarrows \mathrm{S}_{2}^{2-} \\
1 / 2 \mathrm{~S}_{2}^{2-}+\mathrm{e}^{-} & \rightleftarrows \mathrm{S}^{2-} \\
\mathrm{S}^{2-}+2 \mathrm{Li}^{+} & \rightleftarrows \mathrm{Li}_{2} \mathrm{~S}
\end{aligned}
$$

All reactions are assumed to take place at interfaces (sulfur/electrolyte, carbon/electrolyte, and lithium sulfide/ electrolyte). Note that the mechanism represents a linear chain of reactions. Therefore all sulfur can and will be converted to $\mathrm{Li}_{2} \mathrm{~S}$ during a complete discharge. No side or degradation reactions are considered in this work.

\subsection{Governing equations and model parameters}

The modeling framework has been presented in detail in Ref. [13] and is only summarized here. All model equations are given in Table 1 . In the liquid electrolyte, multicomponent mass and charge transport is described by dilute solution theory under the assumption of charge neutrality. Current is produced by Faradaic reactions (chargetransfer reactions) as well as charge/discharge of an electrochemical double layer at the electrodes' surfaces. A multi-phase management is included, modeling the spatiotemporal evolution of all bulk volume fractions. Changing solid volume fractions affects the effective diffusion coefficients and specific surface areas, thereby altering transport and chemical properties.

Electrochemistry is described with an elementary kinetic approach (no Butler-Volmer equation, no Nernst equation, cf. [15]). As no information on temperaturedependent kinetic coefficients is available, the model is assumed isothermal, and the activation energies are assumed to be zero for all reactions. Note that for most reactions, we specify the kinetic coefficients for forward and reverse reaction separately; the model is therefore not thermodynamically consistent. A consistent model would require the specification of the thermodynamic properties (enthalpies, entropies) of all intermediate species, which are not known at the present time.

All calculations are performed at room temperature and ambient pressure $(T=298 \mathrm{~K}, p=101325 \mathrm{~Pa})$. For all solid species, the activity is equal to 1 . Table 2 contains a full list of the parameters used for the simulation. Parameters are either converted from Kumaresan et al. [12] or assumed. The validation of the model and its underlying parameters using experimental data is subject of ongoing studies.

\subsection{Simulation methodology}

Simulations are carried out using the in-house software package DENIS (detailed electrochemistry and numerical impedance simulation) $[13,16]$. The $1 \mathrm{D}$ computational domain is discretized into 15 compartments using a finitevolume method. Computational time for a discharge curve is about $1 \mathrm{~min}$. on a standard desktop computer. For the cycling simulation, a constant current (CC) discharge is simulated, followed by a constant current/constant voltage (CCCV) charge. Note that, unless the charging current is very small, it was not possible to simulate a full recharge at a constant current due to strongly increasing cell voltage, resulting in numerical instability. Instead, a CCCV charge cycle was simulated: when the cell voltage exceeds $2.8 \mathrm{~V}$ during charging, the cell is held at that voltage until the current drops below $1 \%$ of the original charging current. Similar numerical instabilities occur at the end of the CC discharge, where the simulation terminates for numerical reasons when the reactant concentrations become too low, typically at a cell voltage approximately $5 \%$ below the plateau voltage.

For the impedance simulation, a voltage step/current relaxation technique is used [15]. Starting from opencircuit voltage $(\mathrm{OCV})$, the voltage is increased by $1 \mathrm{mV}$ within $0.1 \mu \mathrm{s}$ and the resulting current transient is simulated over $1000 \mathrm{~s}$. The resulting time traces of voltage and current are Fourier-transformed in the range of $10^{7}$ to $10^{-3} \mathrm{~Hz}$ in order to obtain the complex impedance $Z$ in the frequency domain. The simulation of an impedance spectrum takes around 5 min on a standard desktop computer. Note that the impedance simulation is based on the full physicochemical model, including detailed chemistry and transport, without the use of equivalent circuits.

\section{Results}

\subsection{Discharge at different $C$ rates}

Simulated discharge behavior of the cell is shown in Fig. 2 for different $\mathrm{C}$ rates, i.e. different discharge currents. Fig. 2a presents voltage-capacity curves. They show the typical three-stage behavior also known from experiments [4, 17], consisting of (I) a high-voltage plateau, followed by (II) a fast voltage drop (at low currents associated with a voltage dip), and finally (III) a long low-voltage plateau. 
The origin of this curve shape will be discussed below. The rate capability of the simulated cell is not very good (considerable voltage drop at increasing current), which is typical of $\mathrm{Li} / \mathrm{S}$ batteries with high sulfur content. For all C-rates, $\mathrm{S}_{8}$ is almost completely converted to $\mathrm{Li}_{2} \mathrm{~S}$ (close to $100 \%$ sulfur utilization). The predicted energy density is $1300 \mathrm{Whl}^{-1}$ and $850 \mathrm{Wh} \mathrm{kg}^{-1}$ (per cathode volume/weight). Experimentally, lower values are typically observed due to side reactions or passivation of the cathode (e.g. by film formation); these effects are neglected in this model.

One of the most interesting aspects of the $\mathrm{Li} / \mathrm{S}$ cell is the dissolution and re-formation of phases during cycling. Fig. $2 \mathrm{~b}$ shows the volume fractions of solid $\mathrm{S}_{8}$ and $\mathrm{Li}_{2} \mathrm{~S}$ during discharge. During stage I, solid $\mathrm{S}_{8}$ is completely dissolved. During stage II, all intermediates are present in dissolved form only. Finally, during stage III, $\mathrm{Li}_{2} \mathrm{~S}$ is formed and continuously precipitated. This behavior is observed for all C-rates investigated, i.e. there is no coexistence of solid $\mathrm{S}_{8}$ and $\mathrm{Li}_{2} \mathrm{~S}$ phases during the initial discharge. Another characteristic feature of the $\mathrm{Li} / \mathrm{S}$ cell is the difference in the total solid volume between the charged and discharged state: The volume of the product $\left(\mathrm{Li}_{2} \mathrm{~S}\right)$ is, in fact, $176 \%$ that of the reactant $\left(\mathrm{S}_{8}\right)$ due to the large difference in density of the two phases (cf. Table 2).

Fig. 2c shows spatially averaged concentrations of dissolved $\mathrm{S}_{8(\mathrm{ds})}$ and $\mathrm{S}^{2-}$ species as representative intermediates. Using these profiles, the particular shape of the discharge behavior can be further interpreted. During the plateau stages I and III, the solid $\mathrm{S}_{8}$ and $\mathrm{Li}_{2} \mathrm{~S}$ phases are in equilibrium with the corresponding dissolution product $\mathrm{S}_{8(\mathrm{ds})}$ and $\mathrm{S}^{2-}$ respectively, buffering its concentration to a relatively constant value and thereby causing a cell voltage plateau. This is the typical behavior of phase conversion electrodes. During the intermediate stage II, however, there are no solid phases present and the complete electrochemistry occurs among the electrolyte-dissolved polysulfide intermediates. Within stage II, the concentration of $\mathrm{S}_{8(\mathrm{ds})}$ strongly decreases, while the concentration of $\mathrm{S}^{2-}$ shows a peak. The cell voltage qualitatively follows the $\mathrm{S}_{8(\mathrm{ds})}$ concentration profile, including the respective dip in voltage and concentration at low C-rates, which demonstrates the dominating role of the $\mathrm{S}_{8(\mathrm{ds})}$ species for macroscopic discharge behavior. An interesting effect can be observed for the slower discharge rates: For the polysulfide concentrations to stabilize, it is required that the activity of the solid product be constant. As long as there is virtually no solid $\mathrm{Li}_{2} \mathrm{~S}$ in the cell, this is not the case: The more precipitate is formed, the higher its activity. Because the $\mathrm{Li}_{2} \mathrm{~S}$ precipitation reaction is not an electrochemical reaction, as a first approximation the rate of $\mathrm{Li}_{2} \mathrm{~S}$ formation is independent of the current applied. The voltage profile will only show the dip if no solid $\mathrm{Li}_{2} \mathrm{~S}$ is present at the beginning of the discharge and if the discharge rate is slow compared to the rate of $\mathrm{Li}_{2} \mathrm{~S}$ precipitation.

\subsection{Cycling}

Simulated cycling behavior is shown in Fig. 3. In panels a) and b) the transient cell voltage and current are plotted for the duration of the numerical experiment, consisting of CC discharge, CCCV recharge, and relaxation to OCV. Note that the model shows quite high charging overpotentials, which become particularly obvious at the end of the CCCV phase, when the relaxation of the cell to OCV is accompanied by a strong drop in voltage.

Fig. 3c shows the volume fractions of the bulk reactant and product phases in the composite cathode. The model predicts an asymmetric behavior during cycling: While $\mathrm{Li}_{2} \mathrm{~S}$ is not formed during discharge before all solid $\mathrm{S}_{8}$ is dissolved, the two solid phases do coexist during charge. The reason for this asymmetry is the assumed kinetics of the system in general and the speed of the two dissolution reactions in particular. In case of a partial charge or discharge, this effect can introduce hysteresis to the system.

Fig. 3d shows time traces of the spatially averaged concentrations of all electrolyte-dissolved species. $\mathrm{Li}^{+}$is the only cation present in the cell; due to charge neutrality, the total $\mathrm{Li}^{+}$concentration corresponds to the total concentration of anions in the solution. At the beginning and end of discharge, the non-reactive $\mathrm{PF}_{6}^{-}$is the main counterion, while at intermediate state of charge (SOC), polysulfide anions contribute significantly to the total anion concentration. Both end of charge and end of discharge are characterized by strongly dropping concentrations of dissolved polysulfides (note the logarithmic scale of Fig. 3c), until the reactants for the charge-transfer reactions cannot be supplied anymore. This leads to the final drop of cell voltage at end of discharge, and the final increase in cell voltage at end of charge.

Another interesting result is that polysulfide concentrations are considerably lower during charge compared to discharge. Therefore the conductivity of the electrolyte is lower and diffusion overpotentials are higher during charging. Over the complete cycle, both the total amount and the composition of the ions vary to a great extent. Therefore the electrochemical response of the cell is expected to behave differently at different SOC. This will be further discussed in the following section.

\subsection{Impedance}

The results of impedance simulations for different SOC are shown in Fig. 4. The upper two panels show a Bode representation, i.e. the real and imaginary part of the cell's impedance $Z$ versus logarithmic frequency. The lower panel shows the same information in Nyquist representation, i.e. imaginary part versus real part. The cell shows a complicated impedance behavior, which strongly depends on the SOC. At high SOC, a feature at $\sim 0.5 \mathrm{~Hz}$ is dominating, with an additional smaller feature at $\sim 10 \mathrm{~Hz}$. At increasing SOC, these features merge at an intermediate frequency. Additionally, impedance strongly increases toward low frequencies $<10 \mathrm{mHz}$, which is typical of batteries. 


\section{Conclusions \& Outlook}

A one-dimensional continuum model of a $\mathrm{Li} / \mathrm{S}$ cell with detailed electrochemistry was used to predict voltage, current, capacity, impedance, bulk volume fractions, and dissolved species concentrations under various operating conditions. The simulations show that the discharge behavior of the $\mathrm{Li} / \mathrm{S}$ cell is governed by the presence of solid reactant and product phases. The volume fractions of $\mathrm{S}_{8}$ and $\mathrm{Li}_{2} \mathrm{~S}$ in the cathode change considerably during cycling, providing an explanation of the distinct stages during discharge. The model also predicts an asymmetric behavior of phase formation/dissolution when comparing discharge and charge, as well as high charge overpotentials. The predicted electrochemical impedance behavior is complex, showing multiple impedance features.

Although this study demonstrates the feasibility and the potential of physicochemical modeling for understanding $\mathrm{Li} / \mathrm{S}$ electrochemistry, a proper parameterization and validation are key requirements for further conclusions. To this goal, independent experimental measurements of component and cell properties are required, including thermodynamic data of dissolved polysulfides, their diffusion coefficients, specific surface area of the phases, etc. The model can then be compared to macroscopic experimental observables (charge/discharge curves, impedance, ...) for further validation. Parameterized and validated in such a way, simulations can be used for quantitative interpretation of $\mathrm{Li} / \mathrm{S}$ behavior. Subsequent work includes the study of transport phenomena during the shuttle mechanism as well as the optimization of the cell layout in terms of macro- and microstructure. The modeling framework also allows for the addition of side reactions, alternative reaction pathways, and irreversible degradation mechanisms in order to enable simulations of cycle life and cell aging.

[1] J. B. Goodenough, Y. Kim, Challenges for rechargeable batteries, Journal of Power Sources 196 (16) (2011) 6688-6694. doi:10.1016/j.jpowsour.2010.11.074.

[2] B. Scrosati, J. Garche, Lithium batteries: Status, prospects and future, Journal of Power Sources 195 (9) (2010) 2419-2430. doi:10.1016/j.jpowsour.2009.11.048.

[3] P. G. Bruce, S. A. Freunberger, L. J. Hardwick, J.-M. Tarascon, Li-O2 and Li-S batteries with high energy storage, Nature Materials 11 (1) (2012) 19-29. doi: 10.1038/nmat3191.

[4] S. S. Jeong, Y. T. Lim, Y. J. Choi, G. B. Cho, K. W. Kim, H. J. Ahn, K. K. Cho, Electrochemical properties of lithium sulfur cells using PEO polymer electrolytes prepared under three different mixing conditions, Journal of Power Sources 174 (2) (2007) 745750, 13th International Meeting on Lithium Batteries. doi:10.1016/j.jpowsour.2007.06.108.

[5] S. S. Zhang, Effect of discharge cutoff voltage on reversibility of lithium/sulfur batteries with LiNO3contained electrolyte, Journal of The Electrochemical Society 159 (7) (2012) A920-A923. doi:10.1149/ 2.002207 jes.
[6] K. Cai, M.-K. Song, E. J. Cairns, Y. Zhang, Nanostructured Li2S-C composites as cathode material for highenergy lithium/sulfur batteries, Nano Letters 12 (12) (2012) 6474-6479. doi:10.1021/n1303965a.

[7] X. Ji, L. F. Nazar, Advances in Li-S batteries, Journal of Materials Chemistry 20 (2010) 9821-9826. doi:10. 1039/B925751A.

[8] Y. Yang, G. Zheng, S. Misra, J. Nelson, M. F. Toney, Y. Cui, High-capacity micrometer-sized Li2S particles as cathode materials for advanced rechargeable lithium-ion batteries, Journal of the American Chemical Society 134 (37) (2012) 15387-15394. doi:10.1021/ ja3052206.

[9] C. Barchasz, F. Molton, C. Duboc, J.-C. Leprêtre, S. Patoux, F. Alloin, Lithium/sulfur cell discharge mechanism: An original approach for intermediate species identification, Analytical Chemistry 84 (9) (2012) 3973-3980. doi:10.1021/ac2032244.

[10] H.-J. Ahn, K.-W. Kim, A. Jou-Hyeon, G. Cheruvally, Lithium-sulfur, in: J. Garche (Ed.), Encyclopedia of Electrochemical Power Sources, Elsevier, Amsterdam, 2009, pp. 155-161, secondary Batteries - Lithium Rechargeable Systems. doi:10.1016/B978-044452745-5. 00182-9.

[11] B. Scrosati, J. Hassoun, Y.-K. Sun, Lithium-ion batteries. a look into the future, Energy Environ. Sci. 4 (2011) 3287-3295. doi:10.1039/C1EE01388B.

[12] K. Kumaresan, Y. V. Mikhaylik, R. E. White, A mathematical model for a lithium-sulfur cell, Journal of the Electrochemical Society 155 (8) (2008) A576-A582. doi: $10.1149 / 1.2937304$.

[13] J. P. Neidhardt, D. N. Fronczek, T. Jahnke, T. Danner, B. Horstmann, W. G. Bessler, A flexible framework for modeling multiple solid, liquid and gaseous phases in batteries and fuel cells, Journal of the Electrochemical Society 159 (9) (2012) A1-A15. doi:10. $1149 / 2.023209$ jes.

[14] M.-S. Zheng, J.-J. Chen, Q.-F. Dong, The enhanced electrochemical performance of lithium/sulfur battery with protected lithium anode, Advanced Materials Research 476-478 (2012) 676-680. doi:10.4028/ww. scientific.net/AMR.476-478.676.

[15] W. G. Bessler, Rapid impedance modeling via potential step and current relaxation simulations, Journal of the Electrochemical Society 154 (11) (2007) B1186B1191. doi:10.1149/1.2772092.

[16] W. G. Bessler, S. Gewies, M. Vogler, A new framework for physically based modeling of solid oxide fuel cells, Electrochimica Acta 53 (4) (2007) 1782-1800. doi:10. 1016/j.electacta.2007.08.030.

[17] Y. V. Mikhaylik, J. R. Akridge, Low temperature performance of Li/S batteries, Journal of The Electrochemical Society 150 (3) (2003) A306-A311. doi: $10.1149 / 1.1545452$. 
Table 1: Summary of the governing equations of the 1D Li/S model. See [13] for details and a definition of symbols.

Physicochemical process

\section{Charge transport in liquid electrolyte}

Species conservation in liquid electrolyte

Species fluxes

Electroneutrality and charge conservation

\section{Model equation}

$\frac{\partial\left(\varepsilon_{i} c_{i}\right)}{\partial t}=-\frac{\partial J_{i}}{\partial y}+\sum_{m} A_{m}^{V} \dot{s}_{i, m}$

$J_{i}=-D_{i, \mathrm{eff}} \frac{\partial c_{i}}{\partial y}-\frac{z_{i} F}{R T} \cdot c_{i} D_{i, \mathrm{eff}} \frac{\partial \phi_{\mathrm{elyt}}}{\partial y}$

$0=\sum_{i, m} z_{i} F A_{m}^{V} \dot{s}_{i, m}-\sum_{i} z_{i} F \frac{\partial J_{i}}{\partial y}$

\section{Cell current and voltage}

Cell voltage

Total current density (anode and cathode)

$E=\phi_{\text {elde,ca }}-\phi_{\text {elde, an }}$

$i=\int_{y=0}^{l_{\text {electrode }}}\left(i_{F}+i_{d l}\right) d y$

Current density due to electrical double layer (anode and cathode)

$i_{d l}(t)=A_{d l} C_{d l} \frac{\partial(\Delta \phi)}{\partial t}$

Faradaic current density

$i_{F}=\sum_{m} F A_{m}^{V} \dot{s}_{\text {electron }, m}$

Potential step (anode and cathode)

$\Delta \phi=\phi_{\text {elde }}-\phi_{\text {elyt }}$

\section{Electrochemistry}

Rate equations

$\dot{s}_{i}=\nu_{i}\left(k_{\mathrm{fwd}} \prod_{j} a_{j}^{\nu_{j}^{\prime}}-k_{\mathrm{rev}} \prod_{j} a_{j}^{\nu_{j}^{\prime \prime}}\right)$

Arrhenius rate law (forward and reverse reactions)

$k=k_{0} \exp \left(-\frac{E^{\text {act }}}{R T}\right) \exp \left(-\frac{\alpha z F}{R T} \Delta \phi\right)$

\section{Multi-phase management (cathode)}

Continuity equation for bulk phases

$\frac{\partial\left(\rho_{i} \varepsilon_{i}\right)}{\partial t}=M_{i} \cdot \sum_{m} A_{m}^{V} \dot{s}_{i, m}$ with $\sum_{i} \varepsilon_{i}=1$

Dependence of diffusion coefficients on bulk phases

$D_{i, \mathrm{eff}}=D_{i} \cdot \varepsilon_{i} / \tau_{i}^{2}$

Dependence of specific surface areas on bulk phases

$A^{V}=A_{0}^{V} \cdot\left(\varepsilon_{i} / \varepsilon_{i, 0}\right)^{1.5}$ 


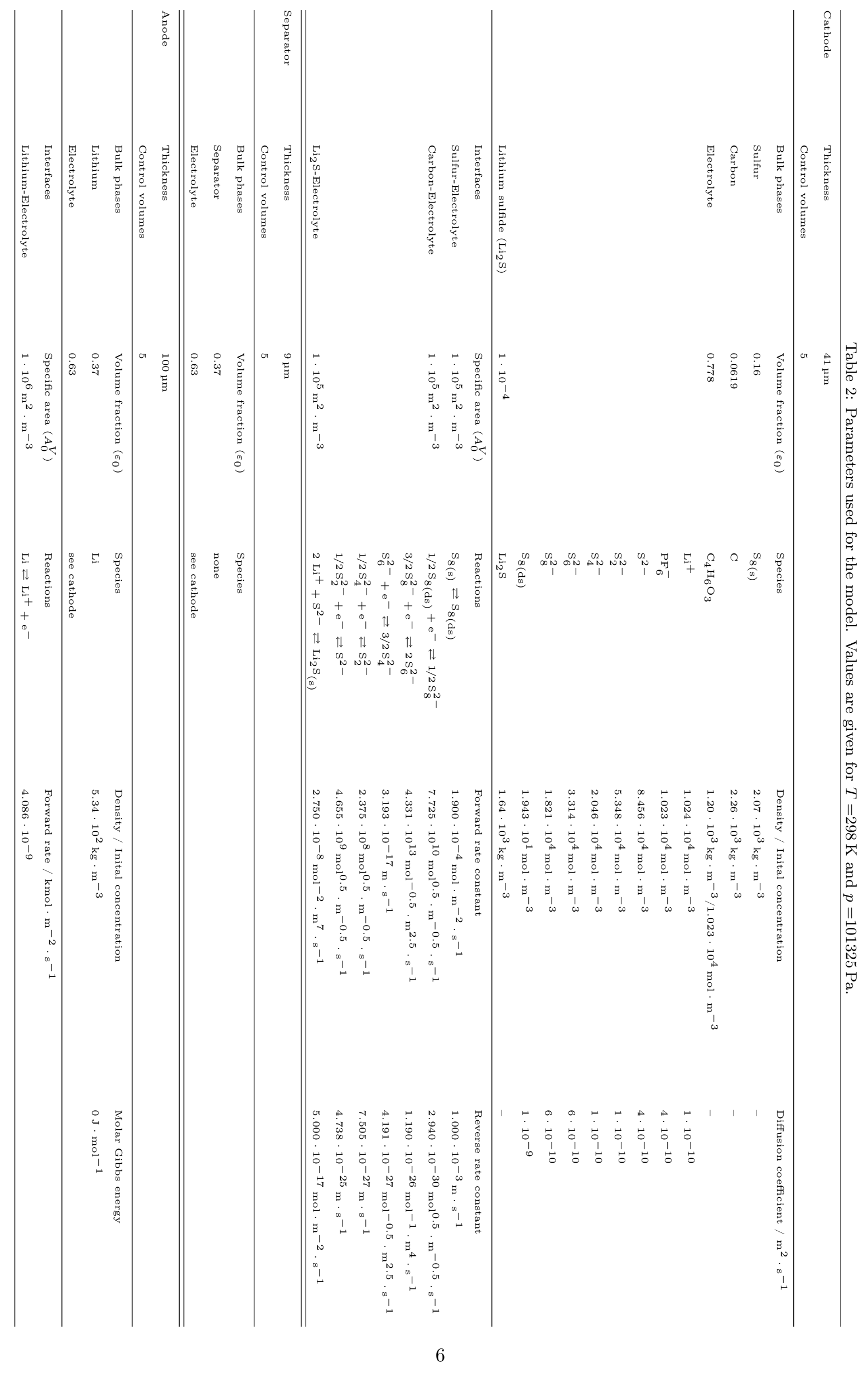




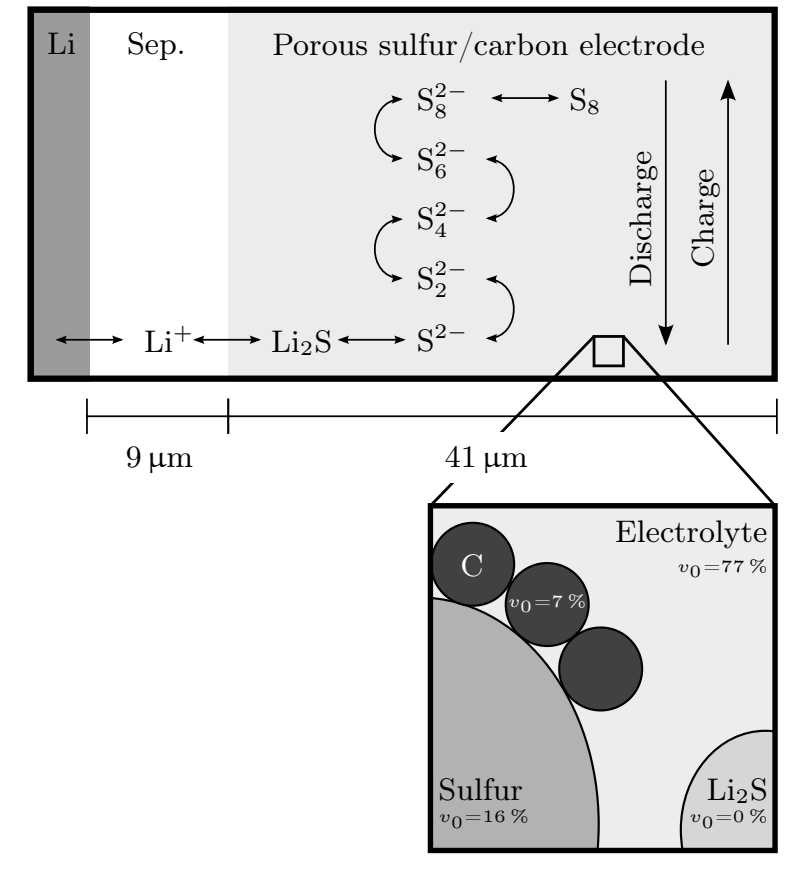

Figure 1: Layout and initial cathode composition of the simulated $\mathrm{Li} / \mathrm{S}$ cell. a)

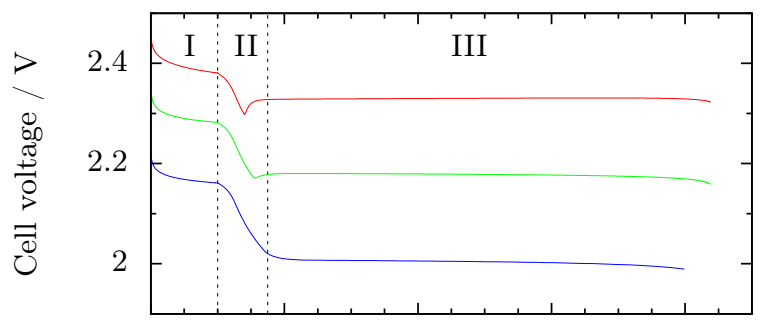

b)
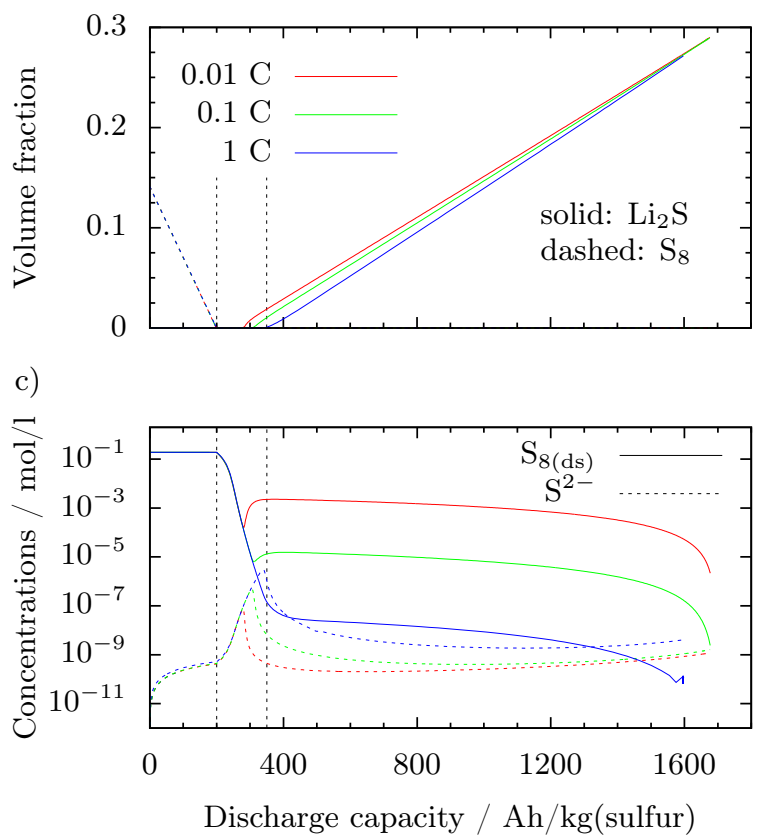

Figure 2: Simulated discharge at various current rates: a) Cell voltage, b) solid volume fractions of $\mathrm{S}_{8}$ and $\mathrm{Li}_{2} \mathrm{~S}, \mathrm{c}$ ) spatially averaged concentrations of selected polyanion species. 


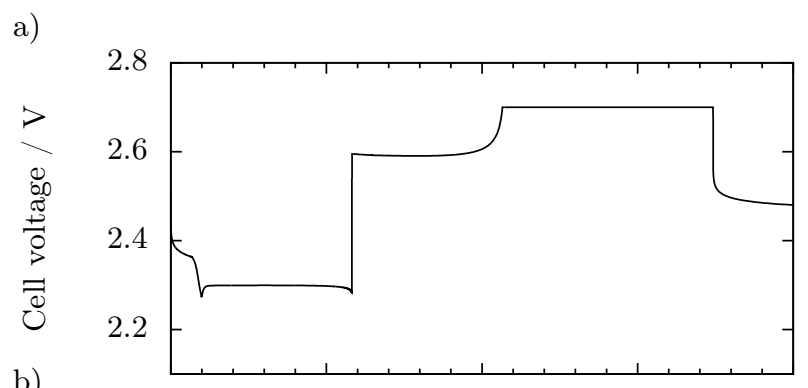

b)
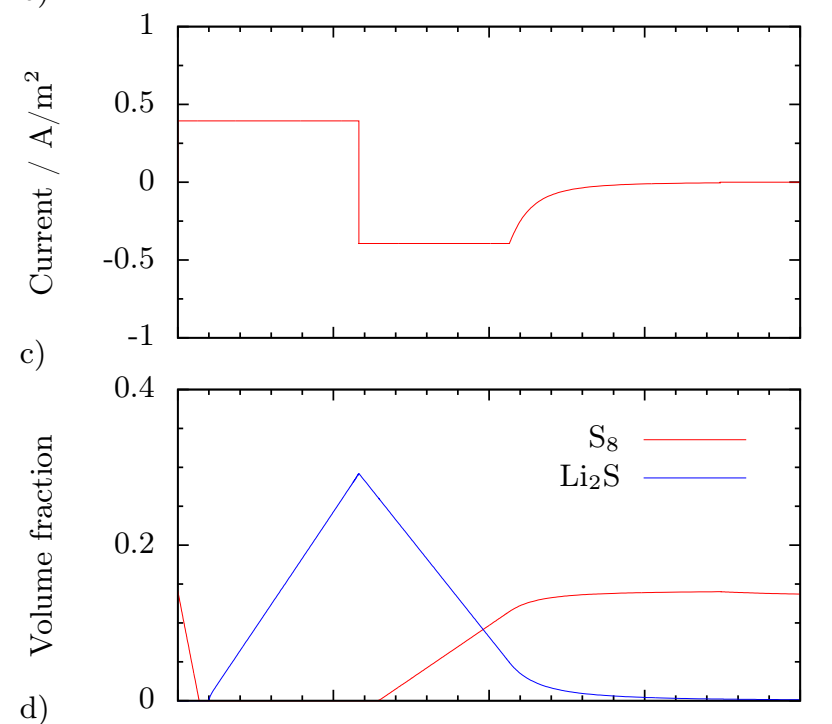

d)

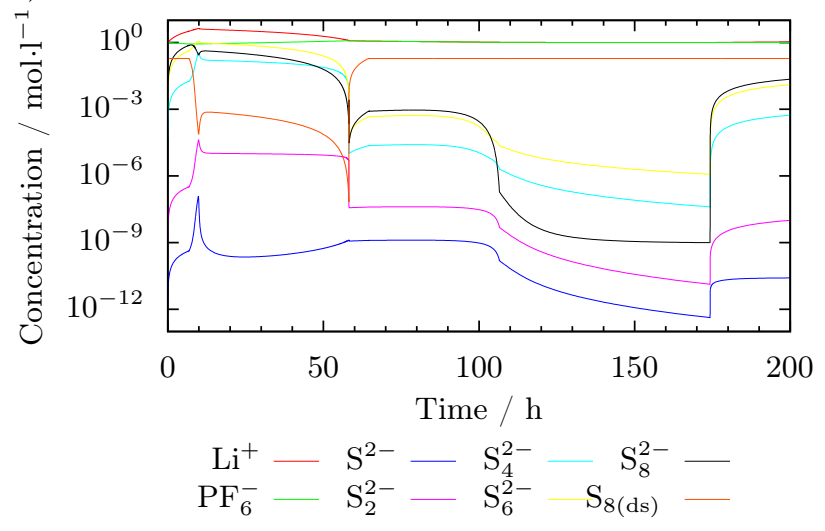

Figure 3: Simulated cycling with CC discharge and CCCV charge at $\mathrm{C} / 50$ rate. a) Cell voltage, b) current, c) Volume fractions, d) spatially averaged concentrations of dissolved intermediates.
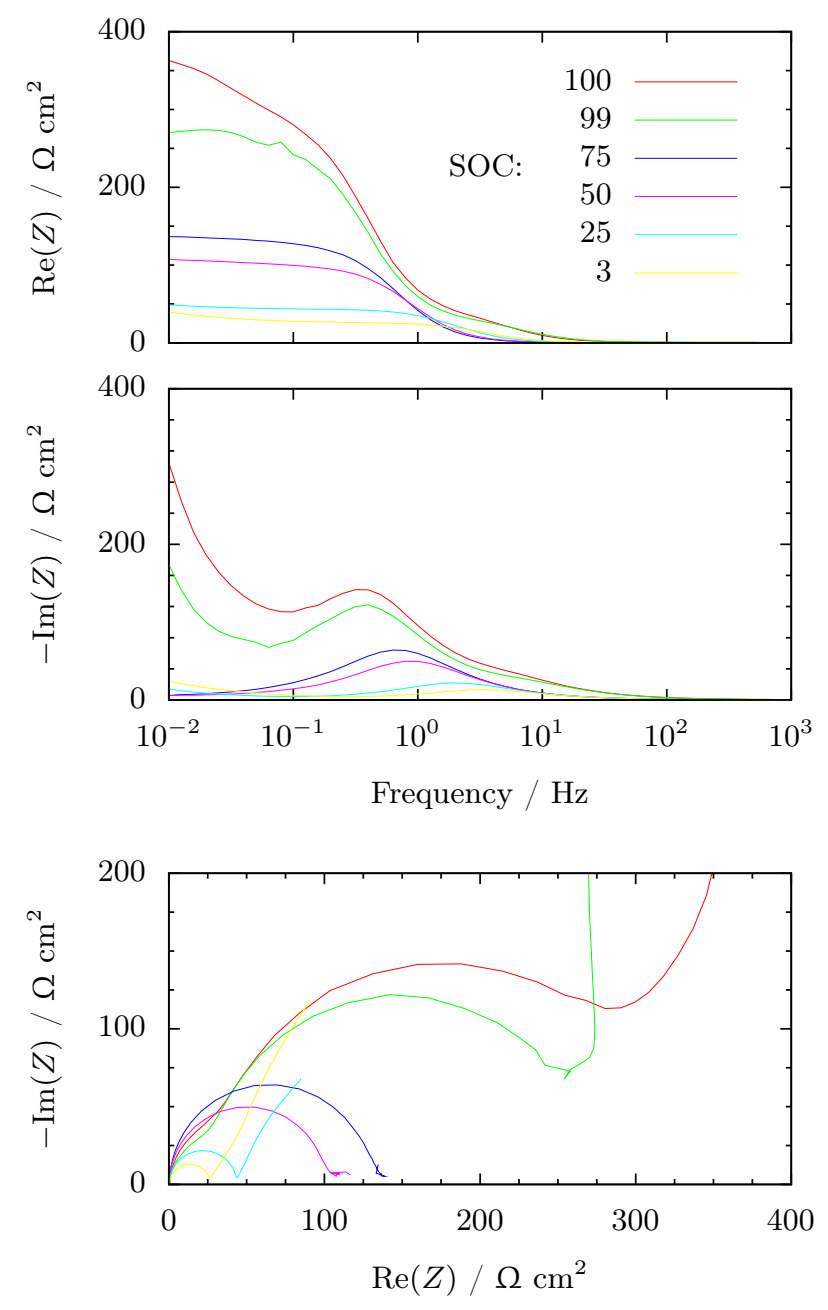

Figure 4: Simulated electrochemical impedance spectra for different SOC. Upper panels: Bode representation. Lower panel: Nyquist representation. 